\section{POS1009 ASYMPTOMATIC ENTHESITIS AND DOMINANT SIDE RELATED PATTERNS IN SPONDYLOARTHRITIS - THE ROLE OF ULTRASONOGRAPHY IN EVERYDAY PRACTICE}

R. Decianu ${ }^{1}$, V. Bojinca ${ }^{2,3}$, L. Groseanu ${ }^{2,3}$, S. Daia-lliescu ${ }^{2,3}$, A. Borangiu ${ }^{2,3}$, C. L. Constantinescu, ${ }^{2,3}$, C. Cobilinschi ${ }^{2,3}$, M. Duna ${ }^{2}$, I. R. Gheorghe ${ }^{4}$, D. Mazilu ${ }^{2,3}$, M. M. Negru, 2,3, D. Opris-Belinski, ${ }^{2,3}$, I. Saulescu ${ }^{2,3}$, O. Vutcanu ${ }^{5}$, A. Balanescu, ${ }^{2,3}$ M. Bojinca ${ }^{3,5}$, R. Ionescu ${ }^{2,3} .{ }^{1}$ National Institute of Rehabilitation, Physical Medicine and Balneoclimatology, III, Bucharest, Romania; " "Sfanta Maria" Clinical Hospital, Internal Medicine and Rheumatology, Bucharest, Romania; 3"Carol Davila"University of Medicine and Pharmacy, Rheumatology, Bucharest, Romania; "'Carol Davila"University of Medicine and Pharmacy, Marketing and Medical Technology, Bucharest, Romania; " "Dr. I. Cantacuzino" Clinical Hospital, Internal Medicine and Rheumatology, Bucharest, Romania

Background: The spondyloarthritis (SpA) family includes a heterogeneous spectre of inflammatory diseases with several common features, such as the axial and peripheral joint involvement, but also trigger factors, as for instance, mechanical stress, infectious diseases or gut dysbiosis.[1,2]

Entheseal inflammation is the common background in the pathogenesis of SpA, leading to osteitis, periostitis and osteoproliferation. Despite being a central feature in $\mathrm{SpA}$, enthesitis remains underdiagnosed in everyday practice.[3,4] Objectives: The primary objective is to assess the frequency of ultrasound versus clinically detected enthesitis in a sample of Romanian patients with highly active SpA. A secondary objective is to determine the existence of an enthesitis pattern according to the dominant side in this sample of patients.

Methods: Out of $140 \mathrm{SpA}$ patients, 106 were diagnosed with axial/peripheral SpA and 34 with psoriatic arthritis (PsA), and were clinically (medical history, clinical examination) evaluated, scanned using MSUS during the same visit, then asked which was their dominant side, in order to avoid any biases. The evaluation targeted 16 entheseal sites (Achilles tendon, plantar fascia, quadriceps tendon, proximal and distal patellar tendon, triceps tendon, extensor and flexor tendons of the hand, all evaluated on both sides), reaching a total of 2240 entheses. The medical history form included questions related to present or past spontaneous pain in any of the 16 evaluated sites as well as the clinical examination that evaluated the entheseal pain upon pressure (digital pressure on the enthesis overlying skin).

The MSUS evaluations were conducted using Esaote My Lab machines with 6-12/8-18 MHz linear probes. The same clinician/ultrasonographer performed all evaluations, in order to avoid interobserver variability.

Results: In the studied sample of patients, $68.6 \%$ were men, had a mean age of 43,46 $(+/-11,77), 51.4 \%$ were diagnosed with peripheral $\mathrm{SpA}$, with a mean disease duration of $61,60(+/-71,03)$ months, and entheseal abnormalities were found in up to $62.7 \%$ of the asymptomatic entheses, lacking both spontaneous and elicited pressure pain. The best performance of clinical and ultrasound examination was observed in the evaluation of the flexor tendons of the hand, with strong agreement between the two methods (kappa $=0,718, p=0.001$ ). Conversely, the lowest performance of clinical and ultrasound examination was noticed in Achilles (kappa $=0,292, p=0.001$ ) and distal patellar tendons (kappa $=0,202, p=0.001$ ), with low agreement indices. Both GS and PD abnormalities were more frequently detected on the right side, in a sample of $98.57 \%$ right handed patients. The differences were higher regarding the insertions of the triceps tendon (2.2\%) and the plantar fascia (2.1\%), in favour of the dominant side. Conclusion: Entheseal reported pain (spontaneous and elicited by pressure) correlates poorly with MSUS detected enthesitis. This study highlights the high rates of imaging detected, but clinically overlooked entheseal abnormalities in SpA patients. Enthesitis was more frequently detected on the dominant side, emphasizing the role of mechanical stress in the pathogenesis of this feature. This outcome also requires the selection of the most reliable entheseal sites for SpA, being a future direction of research for this ongoing study.

REFERENCES:

[1] Barnett R, Ingram T, Sengupta R. Axial spondyloarthritis 10 years on: still looking for the lost tribe. Rheumatology (Oxford). 2020; 59(4): iv25-iv37

[2]Kehl AS, Corr M, Weisman MH, et al. Enthesitis - New Insights Into Pathogenesis, Diagnostic Modalities, and Treatment. Arthritis Rheumatol. 2016; 68(2): 312-322

[3]Ruta S, Gutierrez M, Pena C, et al. Prevalence of subclinical enthesopathy in patients with spondyloarthropathy: an ultrasound study. J Clin Rheumatol. 2011; 17: 18-22

[4] Yi E, Ahuja A, Rajput T, et al. Clinical, economic, and humanistic burden associated with delayed diagnosis of axial spondyloarthritis: a systematic review. Rheumatol Ther. 2020; 7: 65-87

Disclosure of Interests: None declared.

DOI: 10.1136/annrheumdis-2021-eular.3612

\section{POS1010 \\ PREDICTING CARDIOVASCULAR EVENTS IN PATIENTS WITH SPONDYLOARTHRITIS: 3 RISK ALGORITHMS}

A. S. Pinto ${ }^{1,2}$, F. Cunha Santos ${ }^{1}$, S. P. Dinis ${ }^{1}$, F. Guimarães ${ }^{2}$, D. Esperança Almeida $^{3}$, H. Parente ${ }^{2}$ S. Azevedo ${ }^{2}$, C. Vaz ${ }^{1,4}$, D. Faria ${ }^{2}$, J. F. Ferreira ${ }^{1,4}$.

${ }^{1}$ Unidade Local de Saúde da Guarda, Rheumatology, Guarda, Portugal;

${ }^{2}$ Unidade Local de Saúde do Alto Minho, Rheumatology, Ponte de Lima,
Portugal; ${ }^{3}$ Hospital de Braga, Rheumatology, Braga, Portugal; ${ }^{4}$ Universidade da Beira Interior, Faculdade de Ciências da Saúde, Covilhã, Portugal

Background: In the most recent European League Against Rheumatism (EULAR) cardiovascular risk reduction recommendations ${ }^{1}$, the use of the SCORE algorithm has been advocated as a useful tool to identify an increased 10-year cardiovascular risk of first fatal atherosclerotic event. Even though validated inflammatory disease-specific $\mathrm{CV}$ risk score algorithms are still lacking, the EULAR task force advocated the use of a 1.5 multiplication factor for RA, but not clear for other inflammatory diseases.

Objectives: To assess the accuracy of several CV risk algorithms to predict an event and determine its sensibility and specificity.

Methods: A retrospective analysis of Spondyloarthritis $(\mathrm{SpA})$ patients, registered in REUMA.PT, followed in two Portuguese centres was done. We calculated risk prediction algorithms such as Framingham, the American College of Cardiology/ American Heart Association (ACC/AHA) risk score and the Systematic Coronary Risk Evaluation (SCORE) for low-risk countries. The adaptation of risk algorithms was done, accordingly to EULAR recommendations. Primary outcome was the first $\mathrm{CV}$ event. Discriminatory ability for $\mathrm{CV}$ risk prediction was evaluated by the area under the ROC curves. Sensibility and specificity were calculated for low-to-intermediate and intermediate-to-high risk cut-offs. Cut-off values of high risk were defined in $5 \%$ for SCORE, $20 \%$ for Framingham and ACC/AHA.

Results: 362 patients with SpA were included, $53.9 \%$ male (195), with a mean age of $51.1 \pm 12.7$ years. $67.8 \%$ of the patients were HLA B27 positive. Overall, the mean BMI was $26.3 \pm 4.4 \mathrm{Kg} / \mathrm{m}^{2}$ and $24.0 \%$ of the patients (87) were smok ers in their lifetime. The mean of systolic blood pressure was $130 \pm 16.4 \mathrm{mmHg}$, diastolic blood pressure of $73.5 \pm 10.4 \mathrm{mmHg}$, total cholesterol of $190.1 \pm 37.2 \mathrm{mg}$ $\mathrm{dL}$ and high-density lipoprotein cholesterol of $53.0 \pm 14.8 \mathrm{mg} / \mathrm{dL}$. Anti-hypertensive medication was reported in $24.3 \%$ of the patients, cholesterol medication in $19.3 \%$ and antidiabetic medications in $6.1 \%$. Twenty-five patients $(6.9 \%)$ presented a cardiovascular event. Patients with a cardiovascular event were older, with higher $\mathrm{BMI}$, prescribed with medication for $\mathrm{CV}$ comorbidities and higher diastolic and systolic blood pressure $(p<0.05)$

Patients were under biologic therapy in $30.9 \%$ (112), 16.9\% (61) with methotrexate; $16.3 \%(59)$ with sulfasalazine and $2.8 \%$ (10) with leflunomide; $68.5 \%$ (248) prescribed with NSAID and $10.8 \%$ (39) with corticosteroids. Area under the ROC in original and adapted scores were equal: 0.709 (95\% Cl 0.598 to 0.819$)$ for SCORE, $0.805(95 \% \mathrm{Cl} 0.737$ to 0.872$)$ for Framingham and $0.776(95 \% \mathrm{Cl}$ 0.695 to 0.857 ) for ACC/AHA (Figure 1).
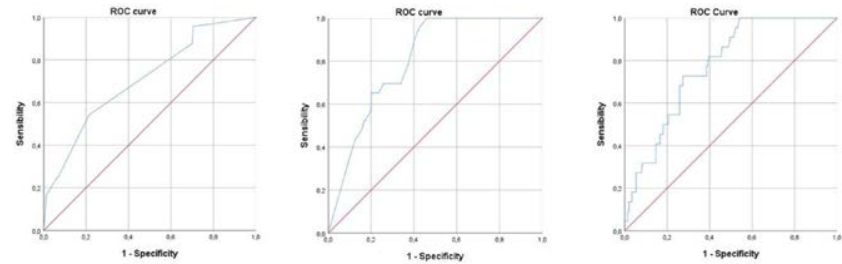

Figure 1. ROC curves for SCORE, Framingham and ACC/AHA

SCORE $>1 \%$ showed the best sensitivity $(96 \%)$ but lower specificity. Framing ham $>20 \%$ presented the best specificity $(80 \%)$ with lower sensitivity $(61 \%)$. In all cases, specificity raises with higher cut-off with corresponding reduction in sensibility. (Table 1)

Table 1. Sensibility, specificity, positive predictive value (PPV) and negative predictive value (NPV) of CV risk algorithms

\begin{tabular}{llllll}
\hline & \multicolumn{2}{c}{ Sensibility (\%) } & Specificity (\%) & PPV (\%) & NPV (\%) \\
\hline SCORE $>1 \%$ & 96 & 29 & 12 & 99 \\
SCORE $>5 \%$ & 54 & 78 & 20 & 94 \\
Framingham $>10 \%$ & 78 & 63 & 15 & 97 \\
Framingham $>20 \%$ & 61 & 80 & 21 & 96 \\
ACC/AHA $>5 \%$ & 82 & 55 & 16 & 97 \\
ACC/AHA $>20 \%$ & 32 & 86 & 20 & 92
\end{tabular}

Conclusion: A good discrimination between patients with or without CV events has been demonstrated by area under the $\mathrm{ROC}$ curve. The adaptation of $\mathrm{CV}$ risk algorithms according EULAR recommendations did not provide an improvement in discriminative ability. Overall, the algorithms studied presented a low sensibility or specificity. Better algorithms are needed to correctly assess cardiovascular risk for SpA patients and they should take into consideration the risk associated with the disease.

\section{REFERENCES:}

[1] Agca R, Heslinga SC, Rollefstad S, et al. EULAR recommendations for cardiovascular disease risk management in patients with rheumatoid arthritis and other forms of inflammatory joint disorders: 2015/2016 update. Annals of the Rheumatic Diseases 2017;76:17-28.

Disclosure of Interests: None declared.

DOI: 10.1136/annrheumdis-2021-eular.3622 